\title{
Research highlights from the 2018 ERS International Congress: interstitial lung diseases
}

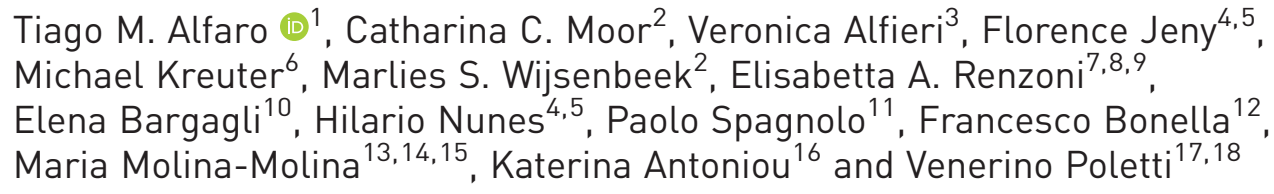

Affiliations: ${ }^{1}$ Unit of Respiratory Medicine A, Centro Hospitalar e Universitário de Coimbra, Coimbra, Portugal. ${ }^{2}$ Dept of Respiratory Medicine, Erasmus Medical Center, University Hospital Rotterdam, Rotterdam, The Netherlands. ${ }^{3}$ Respiratory Disease and Lung Function Unit, Dept of Medicine and Surgery, University of Parma, Parma, Italy. ${ }^{4}$ AP-HP, Pneumology Dept, Avicenne Hospital, Centre de Référence des Maladies Pulmonaires Rares, Bobigny, France. ${ }^{5}$ Paris 13 University, EA2363, Sorbonne Paris Cité, Bobigny, France. ${ }^{6}$ Center for Interstitial and Rare Lung Diseases, Pneumology and Respiratory Critical Care Medicine, Thoraxklinik, University of Heidelberg and Translational Lung Research Center Heidelberg, Member of the German Center for Lung Research, Heidelberg, Germany. ${ }^{7}$ Interstitial Lung Disease Unit, Royal Brompton Hospital, London, UK. ${ }^{8}$ NIHR Clinical Research Facility, Royal Brompton Hospital, London, UK. ${ }^{9}$ Fibrosis Research Group, Inflammation Repair and Development Section, Imperial College London, London, UK. ${ }^{10}$ Section of Respiratory Diseases and Lung Transplantation, Dept of Clinical Medicine and Neurosciences, Siena University Hospital, Siena, Italy. ${ }^{11}$ Section of Respiratory Diseases, Dept of Cardiac, Thoracic, Vascular Sciences and Public Health, University of Padova, Padua, Italy. ${ }^{12}$ Interstitial and Rare Lung Disease Unit, Dept of Pulmonary Medicine, University Hospital - Ruhrlandklinik, Essen, Germany. ${ }^{13}$ Dept of Pneumology, Bellvitge University Hospital, Barcelona, Spain. ${ }^{14}$ Pneumology Research Group, IDIBELL, University of Barcelona, Barcelona, Spain. ${ }^{15}$ Research Network in Respiratory Diseases (CIBERES), ISCIII, Madrid, Spain. ${ }^{16}$ Dept of Thoracic Medicine, Heraklion University Hospital, Medical School, University of Crete, Heraklion, Greece. ${ }^{17}$ Dept of Diseases of the Thorax, Ospedale GB Morgagni, Forli, Italy. ${ }^{18}$ Dept of Respiratory Diseases and Allergy, Aarhus University Hospital, Aarhus, Denmark.

Correspondence: Tiago Alfaro, Serviço de Pneumologia, CHUC, Praceta Mota Pinto, 3000-075 Coimbra, Portugal. E-mail: alfarotmagmail.com

ABSTRACT This article reviews a selection of the scientific presentations on interstitial lung disease (ILD)/diffuse parenchymal lung disease (DPLD) that were made at the 2018 European Respiratory Society (ERS) International Congress in Paris. A number of advances in the epidemiology, pathogenesis, diagnosis and treatment of these disorders were presented and discussed by clinicians and researchers. The research topics span over all four groups of ERS Assembly 12: Interstitial Lung Diseases (Group 12.01: Idiopathic interstitial pneumonias; Group 12.02: ILD/DPLD of known origin; Group 12.03: Sarcoidosis and other granulomatous ILD/DPLD; Group 12.04: Rare ILD/DPLD).

@ERSpublications

A selection of the scientific presentations on interstitial lung disease from the 2018 \#ERSCongress in Paris http://ow.ly/LAbD30nmsFs

Cite this article as: Alfaro TM, Moor CC, Alfieri V, et al. Research highlights from the 2018 ERS International Congress: interstitial lung diseases. ERJ Open Res 2019; 5: 00215-2018 [https://doi.org/ 10.1183/23120541.00215-2018].

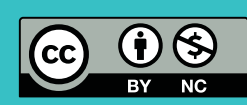

\section{Received: Nov 152018 | Accepted: Dec 262018}

Copyright $\odot$ ERS 2019. This article is open access and distributed under the terms of the Creative Commons Attribution Non-Commercial Licence 4.0. 


\section{Introduction}

This article reviews a selection of the scientific presentations on interstitial lung disease (ILD)/diffuse parenchymal lung disease (DPLD) that were made at the 2018 European Respiratory Society (ERS) International Congress in Paris. A number of topics were presented and discussed by clinicians and researchers. They included new developments in epidemiology, pathogenesis, diagnosis and treatment of these disorders. The subjects of the presentations span over all four groups of ERS Assembly 12: Interstitial Lung Diseases (Group 12.01: Idiopathic interstitial pneumonias; Group 12.02: ILD/DPLD of known origin; Group 12.03: Sarcoidosis and other granulomatous ILD/DPLD; Group 12.04: Rare ILD/DPLD).

\section{Group 12.01: Idiopathic interstitial pneumonias}

A wide variety of basic, translational and clinical research in idiopathic interstitial pneumonias was presented at the 2018 ERS International Congress. Most studies in Group 12.01 focused on idiopathic pulmonary fibrosis (IPF) or combined the different ILDs.

Several new therapeutic targets for IPF were presented. Chitotriosidase is upregulated in lungs of patients with ILDs, including IPF. The selective chitinase inhibitor OATD-01 reduced lung fibrosis in a mouse model and had an acceptable safety profile in healthy volunteers [1,2]. WolfFs et al. [3] showed that the calcium-sensing receptor (CaSR) is upregulated in IPF lungs and CaSR antagonists reduced fibrotic markers in vitro. A phase 1 study demonstrated an acceptable safety profile of aerosolised pirfenidone in healthy volunteers and IPF patients, with higher concentrations of pirfenidone in epithelial lining fluid and lower plasma levels compared with oral pirfenidone [4]. LeE et al. [5] showed that molecular markers of telomere dysfunction and senescence are similar in lungs from IPF and non-IPF usual interstitial pneumonia (UIP) patterns, which suggests that treatment for IPF might also be effective in other forms of UIP-associated fibrosis.

A number of studies focused on quality of life (QoL) and symptom relief in ILDs. The INSTAGE trial, combining nintedanib and sildenafil in IPF patients with advanced diffusing capacity of the lung for carbon monoxide (DLCO) impairment, showed no significant improvement in health-related QoL [6]. Decline in forced vital capacity (FVC) was numerically lower in patients treated with nintedanib plus sildenafil versus nintedanib alone. In the PRAISE trial, progression of dyspnoea was significantly slowed down in a subgroup of IPF patients by pamrevlumab compared with placebo [7]. Another study suggested that a low dose of morphine was safe for the relief of dyspnoea in patients with ILD [8]. BENDSTRUP et al. [9] showed that the majority of IPF patients were fatigued at diagnosis, based on the Fatigue Assessment Scale (FAS). Treatment with antifibrotic drugs did not influence FAS scores after 3 months. A new home monitoring programme including real-time daily home spirometry was presented. This e-health solution was feasible and highly valued by IPF patients, and enabled early detection of changes in FVC and patient-reported outcomes [10].

The importance of identifying more reliable predictors of disease progression and mortality in IPF is underlined by the large number of studies reporting on predictive factors in this field. A nine-gene bronchoalveolar lavage (BAL) signature derived from BAL transcriptome data was predictive of mortality in IPF and had an added value compared with clinical parameters alone [11]. Nolan et al. [12] showed that the "4-metre gait speed" independently predicted mortality and hospitalisation after 1 year in newly diagnosed patients with IPF, while self-reported frailty was an independent predictor of hospitalisations in fibrotic ILDs [13]. Innovative imaging studies showed that a deep learning algorithm recognised UIP patterns on high-resolution computed tomography (CT) with the same accuracy as thoracic radiologists, that CT-histogram-derived indexes at diagnosis can predict mortality in IPF and that an automated CT quantification tool was able to detect typical changes in patients with an acute exacerbation of IPF [14-16]. Nonetheless, these tools and predictive factors need to be validated in larger patient cohorts.

In recent years, many prospective ILD registries have been established in different countries worldwide and collaborative efforts have led to the initiation of multicountry registries [17, 18]. Data from the EMPIRE registry confirm that there are significant differences in IPF patient characteristics and treatment access between countries [19]. Moreover, findings from an international survey in 41 countries revealed that only $58 \%$ of IPF patients have access to antifibrotic treatment [20]. An international survey of pulmonologists from 66 countries showed that approaches to the diagnosis and treatment of acute exacerbations of IPF also vary widely between countries [21].

Real-life PROOF registry data indicated that pulmonary function remained relatively stable during 24 months in IPF patients treated with pirfenidone [22]. In addition, data from INPULSIS-ON demonstrated that the effects of nintedanib on slowing down FVC decline endured over 192 weeks, with a manageable safety and tolerability profile and without new safety signals [23]. In addition, real-life data showed that the overall bleeding risk in patients with nintedanib was very low [24]. In the European IPF 
Registry, a significant survival difference was found between patients treated with antifibrotic drugs and untreated patients; specifically, $50 \%$ of patients on antifibrotic drugs survived $>100$ months from date of diagnosis [17]. However, it is important to note that the long survival after diagnosis is probably accounted for not only by the effect of antifibrotic drugs, but also by earlier diagnosis of IPF. Other real-life data, from a large observational study on cryobiopsy $(n=699)$, showed that overall mortality was low $(0.3 \%)$ and that the diagnostic yield increased when a higher number of samples was obtained from different lung segments [25]. In two large lung cancer screening studies, 6-7\% of patients had ILD features on low-dose CT [26, 27]. In addition, EzQuibela et al. [27] demonstrated that ILD was an independent risk factor for lung cancer, which was supported by findings from a large retrospective study showing a lung cancer prevalence of $8.2 \%$ among patients with IPF [28].

\section{Group 12.02: ILD/DPLD of known origin}

A large number of presentations focused on ILDs of known origin, with many relating to hypersensitivity pneumonitis, connective tissue disease (CTD)-associated ILD and interstitial pneumonia with autoimmune features (IPAF).

Prognostic factors in hypersensitivity pneumonitis were the focus of a number of presentations, highlighting the clinical need for predictive tools in a disease associated with progressive fibrosis and poor prognosis in a subset of patients. BAL lymphocytosis $>30 \%$ was associated with better survival in a large retrospective study of hypersensitivity pneumonitis patients [29]. Along similar lines, lower BAL lymphocyte counts $(<20 \%)$ were associated with a poor prognosis $[30,31]$. In a small retrospective study, higher lymphocyte counts were associated with response to azathioprine treatment [32]. Comorbidities were also associated with impaired survival $[31,33]$.

The role of genetic predisposition in hypersensitivity pneumonitis was explored. RoLDÁN et al. [34] investigated the association between matrix metalloproteinase (MMP) gene variants (MMP1, MMP2, $M M P 9$ and $M M P 12$ ) and susceptibility to the development of hypersensitivity pneumonitis, stratified according to presence $(n=34)$ or absence $(n=104)$ of autoantibodies. The MMP1 rs7125062 variant was associated with increased risk of hypersensitivity pneumonitis with or without autoantibodies, while the MMP2 rs11646643 genotype was associated with an increased risk for autoantibody-positive hypersensitivity pneumonitis and a worse prognosis, although these findings will need validation. In an exploratory study of 19 patients with hypersensitivity pneumonitis, ŠTERCLOVÁ et al. [35] suggested an association between TOLLIP gene polymorphisms and disease progression, although replication in a larger group of patients is needed.

A focus on predictors of disease behaviour identifiable at baseline was also evident among studies on CTD-ILD. STOск et al. [36] observed that baseline serum KL-6 levels were associated with progression of systemic sclerosis (SSc)-associated ILD in both a retrospective and a prospective validation cohort. In a Spanish cohort, a value of KL-6 $>425 \mathrm{U} \cdot \mathrm{mL}^{-1}$ was reported as the optimal cut-off to differentiate ILD patients (including CTD-ILD and IPF) from healthy controls [37]. In a cohort of 4131 patients with SSc, KREUTER et al. [38] confirmed that ILD is the most frequent type of pulmonary complication, followed by pulmonary hypertension (PH)-ILD and $\mathrm{PH}$ alone, with PH-ILD having the worse survival. In patients with rheumatoid arthritis (RA)-associated ILD, a CT staging system based on the presence of UIP pattern, emphysema and a fibrosis score identified patients with a worse prognosis [39].

In terms of treatment effects, a retrospective analysis of patients with RA-ILD treated ( $\mathrm{n}=26)$ or not $(\mathrm{n}=18)$ with rituximab for joint involvement reported a trend bordering on statistical significance towards a slower rate of lung function decline in the rituximab-treated group [40]. Preliminary data on the effect of nintedanib on fibroblasts from patients with SSc-ILD and control lungs suggest that nintedanib inhibits myofibroblast differentiation and contractility [41].

The significance of autoimmunity features/autoantibodies in ILD was also explored [42, 43]. In a retrospective study of 102 patients with high-resolution CT-defined nonspecific interstitial pneumonia (NSIP) pattern, no differences in clinical characteristics or 3-year survival were observed between patients with IPAF and idiopathic NSIP. However, within the IPAF group, the presence of antisynthetase antibodies was associated with a more frequent acute onset [43]. In a large cohort of 82 anti-MDA5-positive patients, the prevalence of ILD was high $(63 \%)$, with NSIP being the most frequent pattern. Acute onset and rapidly progressive ILD was frequent in this subgroup of patients [44]. Finally, in a Japanese study comparing histological findings between anti-neutrophil cytoplasmic antibody myeloperoxidase-positive $\left(\mathrm{MPO}^{+}\right)$ILD patients $(n=28 ; 20$ with a histology pattern of UIP) and IPF, a greater degree of peri-bronchiolar inflammation was seen surrounding cystic lesions in $\mathrm{MPO}^{+}$ILD, suggesting that the pathogenesis of the cystic changes seen in a UIP pattern associated with $\mathrm{MPO}^{+}$may differ from the honeycomb lesions of IPF [45]. 
The importance of symptom management and improvement of healthcare quality in patients with non-IPF ILD was highlighted by a systematic review on pharmacological and nonpharmacological interventions [46]. In particular, patients with non-IPF progressive fibrosing ILD had higher healthcare utilisation and costs compared with other ILD patients, underlining the need to focus resources in this group [47]. In a large review of the Swedish respiratory failure registry, comprising 1603 ILD patients, the use of low-dose benzodiazepines and the use of either low or high doses of opioids for symptom management of patients with oxygen-dependent ILD appeared safe, since no increased hospital admissions or mortality were reported, whereas an association between high-dose benzodiazepines and mortality was observed [48]. Finally, the inclusion of a specialist pharmacist in the ILD multidisciplinary team is likely to improve the management of drug interactions and adverse effects, optimising treatment adherence and reducing medical costs [49].

A few innovative presentations focused on imaging biomarkers as a tool for detection and quantification of ILD. Magnetic resonance imaging (MRI) findings in animal models of drug-induced ILD were correlated with extent of inflammation and fibrosis $[50,51]$. The use of in vivo confocal laser endomicroscopy during bronchoscopy in nine patients with ground-glass changes on CT provided additional information and appeared to allow differentiation between inflammatory versus fibrotic changes as the underlying cause of ground-glass opacities (partially filled alveoli by cellular infiltrates versus fine fibrosis) [52].

\section{Group 12.03: Sarcoidosis and other granulomatous ILD/DPLD}

The presentations in these sessions almost always concerned the field of sarcoidosis, and provided a rich array of information regarding phenotype, QoL, dangerous sarcoidosis and translational research for understanding disease pathogenesis.

Regarding phenotyping sarcoidosis, Lноте et al. [53] analysed 1237 patients with at least one extrapulmonary localisation. The authors performed a cluster analysis to identify clinical phenotypes. Five clusters were finally identified, in line with those previously reported [54], but in a multiethnic population. In this analysis, phenotypes could be explained, at least partially, by sex, geographical origin and professional environmental exposure.

Schupp et al. [55] presented results from the GenPhenResa study. In this large European cohort including more than 2100 Caucasian sarcoidosis patients, genetic profiles associated to specific phenotypes were studied. Some genetic variants (e.g. polymorphisms) were associated with specific clinical features, such as TNFA rs1800629 with acute sarcoidosis, but links between genetics and phenotype often varied according to the regional origin of patients.

The prognostic value of pulmonary function tests in patients with hypersensitivity pneumonitis has been explored in two large retrospective studies from the Royal Brompton Hospital (London, UK), with decline in FVC $\geqslant 10 \%$ and in DLCO $\geqslant 15 \%$ within the first year both being predictive of mortality after adjusting for age, sex, smoking and exposure history [56]. In pulmonary sarcoidosis, Kouranos et al. [57] analysed the prevalence of mixed ventilatory defect in 1110 patients. Depending upon definition criteria, 25-35\% of sarcoidosis patients with airflow obstruction had a mixed pattern, which was associated with further DLCO reduction compared with patients with only airflow obstruction, and higher prevalence of chest radiographic stage IV than other ventilatory defects (63.5\% for mixed versus $38.3 \%$ for obstructive versus $38.5 \%$ for restrictive defects). Mortality was higher in patients with mixed and restrictive pattern than those with obstruction alone, but this difference was more linked to the level of DLCO than to the type of ventilatory defect per se.

Evaluation and management of QoL impairment are essential in sarcoidosis. An international survey including 1842 patients was undertaken in order to gather views about which treatment outcomes matter most to sarcoidosis patients [58]. QoL and functionality were the highest priority for outcomes of sarcoidosis patients. Blood tests and pulmonary function testing were not viewed as important. In a double-blind, randomised, placebo-controlled trial, the effect of low-dose oral dexamethasone (1 mg) on QoL was studied [59]. A total of 16 patients were randomised and followed-up for 1 year. Low-dose dexamethasone resulted in a reduction of the inflammatory profile, and improved QoL parameters and fatigue, but with higher weight gain than control patients [59].

Cardiac sarcoidosis was the subject of three presentations. OHIRA et al. [60] showed that the prevalence of cardiac sarcoidosis diagnosed according to the revised Japanese guidelines [61] exceeds 20\% in biopsy-proven extracardiac sarcoidosis patients with no cardiac symptoms, and normal ECG and echocardiogram. This raises the question of systematic screening with MRI and positron emission tomography in this particular population. In a study from Poland, lower left ventricular ejection fraction was associated with decreased value of forced expiratory volume in $1 \mathrm{~s}(\mathrm{FEV} 1)(\mathrm{r}=0.31, \mathrm{p}=0.003)$ in cardiac sarcoidosis [62]. The main hypothesis is that heart failure may cause bronchial wall oedema [63]. An 
alternative hypothesis is the existence of a phenotype associating obstruction and cardiac sarcoidosis. Nevertheless, a decrease of FEV1 should warn of the possibility of heart failure in sarcoidosis. The Royal Brompton Hospital [64] reported its large experience of 644 patients referred for suspected cardiac sarcoidosis. Cardiac sarcoidosis diagnosis was based on Heart Rhythm Society consensus statement criteria [65] via multidisciplinary team discussion. Two groups were identified: one with known extracardiac sarcoidosis $(n=461)$ and one with cardiac manifestation as first presentation $(n=183)$. The diagnosis of cardiac sarcoidosis was done in $36.9 \%$ and $76.5 \%$, respectively, of the two groups. Active myocardial inflammation was present in $41.8 \%$ and $60.7 \%$, respectively. Mortality in the first group was $15 \%$ at 10 years and was predicted by late gadolinium enhancement on MRI only in univariate analysis, whereas only age was predictive of mortality in multivariate analysis.

A number of presentations reported on basic research in sarcoidosis. Sснотт et al. [66] studied the role of the immune paradox (i.e. diminished peripheral responses) on peripheral blood mononuclear cells from sarcoidosis patients with a gene network analysis. There was an association between peripheral lymphopenia and worse lung function. Decreased expression of lymphocyte activity genes was observed and associated with a more severe phenotype in sarcoidosis, but also in other ILDs. The importance of T-helper 17 subsets in the pathogenicity and chronicity of sarcoidosis was once again observed [67]. LEPZIEN et al. [68] analysed the distribution of mononuclear phagocytes in different anatomical compartments in patients with Löfgren syndrome and non-Löfgren syndrome sarcoidosis. Cytometry analysis identified different populations of monocytes and dendritic cells among these compartments, but in different proportions. Löfgren syndrome patients had a decreased frequency of dendritic cells in bronchial tissue and lymph nodes, which may translate to differences in T-cell responses associated with disease progression. Mononuclear phagocytes in the bronchial tissue and BAL were more activated than in blood and lung lymph nodes, indicating local inflammation.

Two studies concerning microbiota did not identify a specific profile or pathogen in the lungs of sarcoidosis patients $[69,70]$. Several other lines of research on sarcoidosis were presented: analysis of ubiquitin and PU-1 (a transcriptional activator involved in the differentiation and activation of macrophages) [71], analysis of vascular endothelial growth factor (VEGF) [72], the possible involvement of autoimmune factors, owing to the detection of elevated levels of autoantibodies against modified citrullinated vimentin [73], and metabolomics study on plasma [74].

\section{Group 12.04: Rare ILD/DPLD}

Rare diseases are challenging for both treating physicians and researchers, as they tend to be exposed to a limited number of cases. Most of these conditions are orphan, as they are ultrarare, not widely researched and no effective treatment strategies or approved drugs exist [75]. The 2018 ERS International Congress included several educational and scientific sessions on rare lung diseases, where clinicians and scientists from all around the world shared and discussed new data on the pathogenesis, diagnosis and treatment of these neglected disorders.

Pleuroparenchymal fibroelastosis (PPFE) is a distinctive ILD that may be primary or secondary. Its prognosis is unpredictable, with some cases showing inexorable progression [76]. In a study of 62 lung transplanted patients, 15 had PPFE in pre-transplant imaging studies. Five cases were idiopathic, eight were associated with hypersensitivity pneumonitis and two with IPF [77]. In 72 asymptomatic PPFE subjects, the presence and severity of traction bronchiectasis in PPFE areas was correlated with the extent and severity of the disease $(\mathrm{p}<0.05)$. Plathythorax, with deepened suprasternal notch on CT, correlated with progression $(\mathrm{p}<0.01)$ and death $(\mathrm{p}<0.05)$ [78].

Lymphangioleiomyomatosis (LAM) is a rare cystic lung disease affecting almost exclusively females [79]. Terraneo et al. [80] assessed the levels of serum VEGF-C, VEGF-D, MMP-2 and MMP-7 in 27 LAM patients and 16 healthy volunteers. Higher levels of VEGF-D (area under the curve (AUC) 0.833), MMP-2 (AUC 0.756) and MMP-7 (AUC 0.820) were predictive of LAM diagnosis. NoviKova et al. [81] reported on the use of mammalian target of rapamycin (mTOR) inhibitors in 15 patients with progressive LAM and showed a decrease of abdominal leiomyomas in three patients, with no cases of pneumo-, chylo- or haemothorax, suggesting treatment efficacy.

Pulmonary Langerhans cell histiocytosis (PLCH) is a cystic disorder that is typically associated with smoking [82]. LE GUEN et al. [83] reported on 43 patients with pneumothorax complicating PLCH and found a high risk $(53 \%)$ for recurrence that was not changed by surgery $(\mathrm{p}=0.96)$. Thoracotomy, however, was more effective than video-assisted thoracoscopic surgery in preventing recurrences $(p=0.03)$ and was recommended by the authors [83]. A detailed genotyping analysis of the mitogen-activated protein kinase (MAPK) pathway in $50 \mathrm{PLCH}$ patients found genetic alterations in 44 (88\%). Some of these gene alterations lead to variable sensitivity to MAPK targeting drugs and the authors proposed this strategy for refractory cases [84]. RADZIKOWSKA et al. [85] reported on the effects of cladribine for the treatment of 
PLCH in 12 patients. There was improvement in lung function in five patients and stabilisation in seven patients. None of the patients progressed.

Pulmonary alveolar proteinosis is characterised by alveolar accumulation of surfactant lipids and proteins. INOUE et al. [86] reported on 34 fatal cases from a nationwide Japanese cohort. Deceased patients had a similar age and sex distribution to survivors, but were more symptomatic $(p=0.035)$ and had more frequent secondary disease $(\mathrm{p}<0.001)$. The median survival time for nonsurvivors was 16.5 years post-diagnosis, and major causes of death were malignancy (26.5\%), infection $(20.6 \%)$ and respiratory failure (11.8\%).

In addition to the classical rare DPLDs, there were several reports on ultrarare diseases affecting adults and children. Alimi et al. [87] reported that nine out of 34 patients with pulmonary haemosiderosis had Down syndrome, and this group had more severe disease with increased dyspnoea $(p=0.03)$ and pulmonary arterial hypertension $(\mathrm{p}=0.01)$. Additionally, all the three reported deaths occurred in the Down syndrome group.

MARAngu et al. [88], from Cape Town, South Africa, reported on 12 Zimbabwean children with exogenous lipoid pneumonia caused by repeated oil administration for cultural reasons. Children were between 2.1 and 10.8 months of age, and all displayed cough and alveolar infiltrates on chest radiography. The authors argued for health education for the caregivers and community in order to prevent the disease.

NASSER et al. [89] studied 71 patients with unclassifiable ILD from a national French reference centre and found progressive disease in 46 (64\%). Progressors had higher mortality $(\mathrm{p}=0.004)$, but the only predictor of disease progression was lower baseline FVC (mean \pm SD $70 \pm 20 \%$ versus $84 \pm 27 \%$; $\mathrm{p}=0.049$ ).

Pulmonary lymphangiomatosis is an extremely rare disease characterised by lung, pleural and mediastinal infiltration by abnormal lymphatics. SARMAND et al. [90] reported on six cases from a tertiary German centre. Most were young adults (mean age 35 years) and five were female. Only one had pulmonary interstitial changes. Three were treated with sirolimus with good response. A 24 -month survival of $83 \%$ was found.

Young et al. [91] reported on a US national registry for childhood ILDs. A total of 254 subjects had been enrolled, of which $23 \%$ were subjected to genetic studies. There was substantial morbidity with failure to thrive in $53 \%$ and use of oxygen at some point in $66 \%$. The most frequent disease was diffuse idiopathic pulmonary neuroendocrine cell hyperplasia. Childhood ILD registries are fundamental for the advancement of research on rare diseases.

TAKEUCHI et al. [92] studied the predictive factors for relapse in 56 consecutive patients with chronic eosinophilic pneumonia. On multivariate analysis, centrilobular infiltrates, but not blood or BAL markers, predicted relapses $(\mathrm{p}=0.032)$. The authors proposed that this finding relates with eosinophilic bronchiolitis.

\section{Concluding remarks}

This article summarises only some of the many and exciting developments on ILD/DPLD that were presented at the 2018 ERS International Congress. We encourage readers to follow-up on their personal topics of interest and aim to spark further interest for participation in the 2019 ERS International Congress in Madrid (https://erscongress.org).

Conflict of interest: T.M. Alfaro reports receiving travel support from Boehringer Ingelheim, Novartis, Astra, Menarini, Mundipharma, Zambon and Roche, and grants from Bayer, outside the submitted work. C.C. Moor has nothing to disclose. V. Alfieri has nothing to disclose. F. Jeny has nothing to disclose. M. Kreuter has nothing to disclose. M.S. Wijsenbeek reports grants and other support from Boehringer Ingelheim and Hoffman la Roche, and other support from Galapagos, outside the submitted work. E.A. Renzoni reports lecture and advisory board fees from Roche and Boehringer Ingelheim, and lecture fees from Mundipharma, outside the submitted work. E. Bargagli has nothing to disclose. H. Nunes reports consultancy and research support fees from Roche/Genentech and Boehringer Ingelheim, and grants and personal fees as a clinical trial investigator from Sanofi and Gilead. P. Spagnolo reports personal fees and nonfinancial support from Roche, Boehringer Ingelheim and Zambon, personal fees from PPM Services, Galapagos and Santhera Pharmaceuticals, outside the submitted work. F. Bonella reports personal fees and nonfinancial support from Roche Pharma, Boehringer Ingelheim and Fujrebio, outside the submitted work. M. Molina-Molina reports grants from Roche, Boehringer Ingelheim and grants from Esteve-Teijin Healthcare, and personal fees for specialised medical training from Chiesi, outside the submitted work. K. Antoniou has nothing to disclose. V. Poletti has nothing to disclose.

\section{References}

1 Dymek B, Sklepkiewicz P, Mlacki M, et al. CHIT1 is a novel therapeutic target in idiopathic pulmonary fibrosis (IPF): anti-fibrotic efficacy of OATD-01, a potent and selective chitinase inhibitor in the mouse model of pulmonary fibrosis. Eur Respir J 2018; 52: Suppl. 62, OA5361. 
2 Lipner J, Lissy M, Dymek B, et al. Phase 1, first-in-human study of OATD-01, a dual chitinase inhibitor for the treatment of respiratory diseases. Eur Respir J 2018; 52: Suppl. 62, PA5228.

3 Wolffs K, Mansfield B, Scofield S, et al. Calcium-sensing receptor as a therapeutic target for pulmonary fibrosis. Eur Respir J 2018; 52: Suppl. 62, OA5365.

4 Montgomery A, Otto K, Khoo J, et al. Late Breaking Abstract - Phase 1 dose escalation study of aerosolized pirfenidone in normal healthy volunteers (NHV), smokers, and IPF patients. Eur Respir J 2018; 52: Suppl. 62, OA266.

5 Lee J, La J, Aziz S, et al. Molecular markers of telomere dysfunction and senescence are common findings in the usual interstitial pneumonia pattern of lung fibrosis. Eur Respir J 2018; 52: Suppl. 62, OA5363.

6 Kolb M, Wells A, Behr J, et al. Nintedanib plus sildenafil in patients with idiopathic pulmonary fibrosis (IPF): the INSTAGE trial. Eur Respir J 2018; 52: Suppl. 62, OA546.

7 Gorina E, Sekayan T, Swigris J, et al. Effect of pamrevlumab on the UCSD-SOBQ (University of California San Diego-Shortness of Breath Questionnaire) in patients with idiopathic pulmonary fibrosis (IPF). Eur Respir J 2018; 52: Suppl. 62, PA5239.

8 Kronborg-White S, Andersen CU, Hilberg O, et al. Safety in treating dyspnea with morphine in ILD patients. Eur Respir J 2018; 52: Suppl. 62, OA5241.

9 Bendstrup E, Skivild V, Larsen R, et al. Fatigue in IPF measured by the Fatigue Assessment Scale during antifibrotic treatment. Eur Respir J 2018; 52: Suppl. 62, PA5237.

10 Moor C, Wapenaar M, Miedema JR, et al. Feasibility of a homemonitoring program including real-time wireless home spirometry in idiopathic pulmonary fibrosis. Eur Respir J 2018; 52: Suppl. 62, PA5232.

11 Prasse A, Carleo A, Jaeger B, et al. BAL cell transcriptome predicts survival in IPF and can be used to gauge and model treatment effects interfering with the TGF-beta pathway. Eur Respir J 2018; 52: Suppl. 62, OA5359.

12 Nolan CM, Maddocks M, Maher TM, et al. Four metre gait speed (4MGS) predicts mortality and hospitalisation in IPF. Eur Respir J 2018; 52: Suppl. 62, PA495.

13 Guler S, Kwan J, Wilcox P, et al. Frailty is an independent predictor of number and length of hospitalizations in patients with fibrotic ILD. Eur Respir J 2018; 52: Suppl. 62, PA1249.

14 Walsh S, Calandriello L, Silva M, et al. A deep learning algorithm for classifying fibrotic lung disease on high resolution computed tomography. Eur Respir J 2018; 52: Suppl. 62, OA262.

15 Torrisi SE, Palmucci S, Rosso R, et al. Assessment of survival in patients with idiopathic pulmonary fibrosis (IPF) using quantitative HRCT indexes. Eur Respir J 2018; 52: Suppl. 62, PA5243.

16 Kehler L, Norajitra T, Ley-Zaporozhan J, et al. Visual and fully-automated CT analysis in acute exacerbations of idiopathic pulmonary fibrosis (IPF). Eur Respir J 2018; 52: Suppl. 62, PA5242.

17 Guenther A, Krauss E, Klepetko W, et al. European IPF Registry: addressing challenges and characteristics of patients with idiopathic pulmonary fibrosis. Eur Respir J 2018; 52: Suppl. 62, PA2198.

18 Vasakova M, Sterclova M, Mogulkoc N, et al. Real world idiopathic pulmonary fibrosis in the EMPIRE registry. Eur Respir J 2018; 52: Suppl. 62, PA2203.

19 Müller V, Šterclová M, Mogulkoc N, et al. Differences in baseline characteristics of newly diagnosed IPF patents in the EMPIRE countries. Eur Respir J 2018; 52: Suppl. 62, PA2204.

20 Wijsenbeek MS, Floricel F, Thonnard J, et al. Idiopathic pulmonary fibrosis - a worldwide review of 'real' life' practice: experience from a treatment feasibility review in 41 countries. Eur Respir J 2018; 52: Suppl. 62, OA263.

21 Kreuter M, Polke M, Walsh S, et al. A global perspective on acute exacerbation of idiopathic pulmonary fibrosis (AE-IPF): results from an international survey. Eur Respir J 2018; 52: Suppl. 62, PA952.

22 Wuyts W, Dahlqvist C, Slabbynck H, et al. Functional decline over time in patients with IPF treated with pirfenidone: the PROOF registry. Eur Respir J 2018; 52: Suppl. 62, PA2201.

23 Crestani B, Quaresma M, Kaye M, et al. Long-term nintedanib treatment in idiopathic pulmonary fibrosis (IPF) final data from INPULSIS-ON. Eur Respir J 2018; 52: Suppl. 62, PA5230.

24 Kolonics-Farkas AM, Šterclová M, Mogulkoc N, et al. Bleeding risk in IPF patients treated with different anticoagulants: real world data from the European MultiPartner IPF Registry (EMPIRE). Eur Respir J 2018; 52: Suppl. 62, PA2205.

25 Ravaglia C, Tomassetti S, Dubini A, et al. Cryobiopsy in the diagnosis of diffuse parenchymal lung diseases: diagnostic strategy and complications in 699 patients. Eur Respir J 2018; 52: Suppl. 62, PA2206.

26 Mikolasch T, Ruparel M, Dickson J, et al. Interstital lung disease rates and risk factors in a UK lung cancer screening trial. Eur Respir J 2018; 52: Suppl. 62, PA2208.

27 Ezquibela AC, Hernandez AM, Gutierrez JG, et al. Interstitial lung disease in a lung cancer screening program: prevalence and association with cancer. Eur Respir J 2018; 52: Suppl. 62, OA566.

28 Tzouvelekis A, Karampitsakos T, Gomatou G, et al. Prevalence of cancer in patients with idiopathic pulmonary fibrosis. A retrospective multicenter study. Eur Respir J 2018; 52: Suppl. 62, PA2908.

29 Wälscher J, Gross B, Bruhwyler J, et al. Prognostic impact of bronchoalveolar lavage lymphocytosis in patients with chronic hypersensitivity pneumonitis. Eur Respir J 2018; 52: Suppl. 62, OA3817.

30 Kim Y, Choe J, Chae EJ, et al. Prognostic factors in Korean patients with chronic hypersensitivity pneumonitis Eur Respir J 2018; 52: Suppl. 62, PA3663.

31 Rittig AH, Hilberg O, Løkke A. Incidence, comorbidity and survival rate of hypersensitivity pneumonitis: a population-based study. Eur Respir J 2018; 52: Suppl. 62, PA3659.

32 Raimundo S, Melo N, Mota P, et al. Factors predicting response to azathioprine in chronic hypersensitivity pneumonitis - the importance of BAL lymphocytosis. Eur Respir J 2018; 52: Suppl. 62, PA2978.

33 Wälscher J, Gross B, Bruhwyler J, et al. Prognostic impact of comorbidities in patients with chronic hypersensitivity pneumonitis. Eur Respir J 2018; 52: Suppl. 62, PA3669.

34 Roldán IB, Ruiz LS, Rubio GP, et al. Genetic variant in MMP2 increases the risk to develop autoantibodies in patients with hypersensitivity pneumonitis. Eur Respir J 2018; 52: Suppl. 62, OA3819.

35 Šterclová M, Petřek M, Kishore A, et al. Effect of genotype on hypersensitivity pneumonitis despite treatment. Eur Respir J 2018; 52: Suppl. 62, PA3665.

36 Stock C, Hoyles R, D'Accord C, et al. Serum KL-6 as a marker of disease progression in SSc-ILD. Eur Respir J 2018; 52: Suppl. 62, PA3664. 

preliminary results from an European cohort. Eur Respir J 2018; 52: Suppl. 62, PA2959. Kreuter M, Bonella F, Blank N, et al. Significance of pulmonary involvement in systemic sclerosis (SSc) - data from the German SSc-network. Eur Respir J 2018; 52: Suppl. 62, PA3657.

39 Song JW, Kim H, Han M, et al. A prognostic staging system for rheumatoid arthritis-associated interstitial lung disease. Eur Respir J 2018; 52: Suppl. 62, PA884.

40 Sra S, Myall K, Lams B, et al. The effect of rituximab treatment on progression of rheumatoid arthritis-associated interstitial lung disease. Eur Respir J 2018; 52: Suppl. 62, OA3821.

41 Bogatkevich G, Atanelishvili I, Akter T, et al. Nintedanib inhibits contractile activity of lung myofibroblasts in a cellular model of scleroderma associated pulmonary fibrosis. Eur Respir J 2018; 52: Suppl. 62, PA3656.

42 Vivarelli E, Vitiello G, Tinghi F, et al. Autoimmunity in interstitial lung diseases: preliminary data from a tertiary center. Eur Respir J 2018; 52: Suppl. 62, PA2991.

43 Biffi A, Dei G, Stainer A, et al. Non specific interstitial pneumonia and features of connective tissue disease: what are the consequences of a different point of view? Eur Respir J 2018; 52: Suppl. 62, PA2972.

44 Meloni F, Cifrian JM, Pesci A, et al. Lung involvement and clinical characteristics in anti-MDA5 positive connective tissue diseases. Eur Respir J 2018; 52: Suppl. 62, PA4215.

45 Tabata E, Takemura T, Baba T, et al. Cystic lesion in myeloperoxidase anti-neutrophil cytoplasmic antibody (MPO-ANCA)-positive interstitial pneumonia: radiological and pathological anaylsis. Eur Respir J 2018; 52: Suppl. 62, OA3820.

46 Bajwah S, Colquitt J, Loveman E, et al. Interventions to improve symptom control and quality of life in patients with interstitial lung disease: a systematic review and meta-analysis. Eur Respir J 2018; 52: Suppl. 62, PA2994.

47 Olson A, Maher T, Salisbury M, et al. Health care resources utilisation and costs in patients with non-IPF progressive fibrosing interstitial lung disease. Eur Respir J 2018; 52: Suppl. 62, PA3658.

48 Bajwah S, Davies J, Tanash $\mathrm{H}$, et al. Safety of benzodiazepines and opioids in interstitial lung disease: national prospective study. Eur Respir J 2018; 52: Suppl. 62, PA5527.

49 Naqvi M, D’Ancona G, West A. Impact of a specialist respiratory pharmacist in the management of interstitial lung disease. Eur Respir J 2018; 52: Suppl. 62, PA3667.

50 Persson IM, Örbom A, Önnervik P-O, et al. Imaging biomarkers of oedema and fibrosis in a rat model of drug-induced-ILD. Eur Respir J 2018; 52: Suppl. 62, PA1958.

51 Persson IM, Håkansson HF, Önnervik P-O, et al. In vivo models of drug induced ILD; tools to study and improve drug safety. Eur Respir J 2018; 52: Suppl. 62, LSC-1058.

52 Wijmans L, Bruin DD, Roelofs J, et al. Confocal laser endomicroscopy (CLE) for differentiating the underlying cause of ground glass opacities in ILD patients. Eur Respir J 2018; 52: Suppl. 62, PA3655.

53 Lhote R, Cohen-Aubart F, Nunes H, et al. Clinical phenotypes of extra-pulmonary sarcoidosis. The EpiSarc study. Eur Respir J 2018; 52: Suppl. 62, OA2159.

54 Schupp JC, Freitag-Wolf S, Bargagli E, et al. Phenotypes of organ involvement in sarcoidosis. Eur Respir J 2018 51: 1700991

55 Schupp J, Freitag-Wolf S, Fischer A, et al. Genetic profiles of clinical features in sarcoidosis. Eur Respir J 2018; 52 Suppl. 62, OA2160.

56 Boccabella C, Macaluso C, Kokosi M, et al. Pulmonary function trends predict mortality in patients with hypersensitivity pneumonitis. Eur Respir J 2018; 52: Suppl. 62, PA4672.

57 Kouranos V, Ward S, Kokosi M, et al. Mixed ventilatory defects in pulmonary sarcoidosis: prevalence and prognosis. Eur Respir J 2018; 52: Suppl. 62, PA5210.

58 Boyd J, Baughman RP, Valeyere D, et al. Sarcoidosis treatment and outcomes: what is most important to patients? Eur Respir J 2018; 52: Suppl. 62, PA5219.

59 Vis R, Van De Garde EMW, Korenromp IHE, et al. Placebo-controlled randomised trial of dexamethasone for quality of life in pulmonary sarcoidosis. Eur Respir J 2018; 52: Suppl. 62, PA5206.

60 Ohira H, Yoshinaga K, Sakiyama S, et al. Risk of underdiagnoses of cardiac sarcoidosis by routine electrocardiogram and echocardiogram in patients with biopsy-proven extracardiac sarcoidosis. Eur Respir J 2018 52: Suppl. 62, OA2154.

61 Terasaki F, Yoshinaga K. New guidelines for diagnosis of cardiac sarcoidosis in Japan. Ann Nucl Cardiol 2017; 3: 42-45.

62 Martusewicz-Boros M, Boros P, Wiatr E, et al. Cardiac sarcoidosis: worse pulmonary function due to left ventricular ejection fraction? A case-control study. Eur Respir J 2018; 52: Suppl. 62, PA5216.

63 Magnussen H, Canepa M, Zambito PE, et al. What can we learn from pulmonary function testing in heart failure? Eur J Heart Fail 2017; 19: 1222-1229.

64 Kouranos V, Sharma R, Khattar R, et al. Cardiac sarcoidosis: a tertiary centre experience. Eur Respir J 2018; 52: Suppl. 62, OA2157.

65 Birnie DH, Sauer WH, Judson MA. Consensus statement on the diagnosis and management of arrhythmias associated with cardiac sarcoidosis. Heart 2016; 102: 411-414.

66 Schott C, Ascoli C, Huang Y, et al. Diminished peripheral T cell activity in sarcoidosis associates with progressive disease. Eur Respir J 2018; 52: Suppl. 62, OA2156.

67 Baranova OP, Ses T, Kudryvzev I, et al. Peripheral blood memory T-helper (Th) 17 subsets in patients with chronic and acute pulmonary sarcoidosis. Eur Respir J 2018; 52: Suppl. 62, OA2158.

68 Lepzien R, Rankin G, Pourazar J, et al. Mononuclear phagocytes in lungs, lymph nodes and blood of sarcoidosis patients. Eur Respir J 2018; 52: Suppl. 62, PA5205.

69 Shaker SB, Durakovic A, Fuursted K, et al. Microbiological study of mediastinal lymph node biopsy from patients with sarcoidosis and lung cancer. Eur Respir J 2018; 52: Suppl. 62, PA5205.

70 Becker A, Vella G, Herr C, et al. The pulmonary microbiome in sarcoidosis is similar to other parenchymal lung diseases. Eur Respir J 2018; 52: Suppl. 62, PA5208.

71 Shin J, Choi BW, Park IW. Differential expression of ubiquitin and PU.1 in granulomatous lesions of tuberculosis and sarcoidosis. Eur Respir J 2018; 52: Suppl. 62, PA5218.

72 Ponomareva L, Popova E, Ponomarev A. The clinical value of vascular endothelial growth factor (VEGF) in the development of lung sarcoidosis. Eur Respir J 2018; 52: Suppl. 62, PA5214. 

2018; 52: Suppl. 62, PA5212.

Terraneo S, Di Marco F, Imeri G, et al. Vascular endothelial growth factors and matrix metalloproteinases serum levels for LAM diagnosis in patients with sporadic LAM and tuberous sclerosis. Eur Respir J 2018; 52: Suppl. 62, OA3785.

81 Novikova LN, Ilkovich M, Dzadzua D, et al. Effectiveness of inhibitor mTOR in patients with lymphangioleiomyomatosis. Eur Respir J 2018; 52: Suppl. 62, PA2240.

82 Vassallo R, Harari S, Tazi A. Current understanding and management of pulmonary Langerhans cell histiocytosis. Thorax 2017; 72: 937-945.

83 Le Guen P, Chevret S, Bugnet E, et al. Pneumothorax in pulmonary Langerhans cell histiocytosis (PLCH). Eur Respir J 2018; 52: Suppl. 62, PA2241.

84 Jouenne F, Lorillon G, Laurent-Issartel C, et al. Genetic landscape of pulmonary Langerhans cell histiocytosis. Eur Respir J 2018; 52: Suppl. 62, PA3539.

85 Radzikowska E, Wiatr E, Blasnska-Przerwa K, et al. Chemotherapy in patients with pulmonary Langerhans cell histiocytosis. Eur Respir J 2018; 52: Suppl. 62, OA3782.

86 Inoue Y, Yamaguchi E, Setoguchi Y, et al. Fatal cases of pulmonary alveolar proteinosis: a nationwide surveillance in Japan. Eur Respir J 2018; 52: Suppl. 62, OA3783.

87 Alimi A, Taytard J, Taam RA, et al. Down syndrome and pulmonary hemosiderosis: an under-recognized association. Eur Respir J 2018; 52: Suppl. 62, PA2237.

88 Marangu D, Pillay K, Banderker E, et al. Exogenous lipoid pneumonia in African children: a mixed-methods case series. Eur Respir J 2018; 52: Suppl. 62, PA2238.

89 Nasser M, Rigaud P, Ahmad K, et al. Unclassifiable interstitial lung disease: a distinct entity with heterogeneous progression. Eur Respir J 2018; 52: Suppl. 62, PA2245.

90 Sarmand N, Piel S, Brunnemer E, et al. Pulmonary lymphangiomatosis - insights into an ultra-rare disease. Eur Respir J 2018; 52: Suppl. 62, PA2246.

91 Young L, Nevel R, Casey A, et al. A national registry for childhood interstitial and diffuse lung diseases in the United States. Eur Respir J 2018; 52: Suppl. 62, OA3786.

92 Takeuchi N, Arai T, Sasaki Y, et al. Relapse predictive factors of chronic eosinophilic pneumonia. Eur Respir J 2018; 52: Suppl. 62, PA3025. 